\title{
Predictability affects the perception of audiovisual synchrony in complex sequences
}

\author{
Laura A. Cook • David L. Van Valkenburg • \\ David R. Badcock
}

Published online: 29 July 2011

(C) Psychonomic Society, Inc. 2011

\begin{abstract}
The ability to make accurate audiovisual synchrony judgments is affected by the "complexity" of the stimuli: We are much better at making judgments when matching single beeps or flashes as opposed to video recordings of speech or music. In the present study, we investigated whether the predictability of sequences affects whether participants report that auditory and visual sequences appear to be temporally coincident. When we reduced their ability to predict both the next pitch in the sequence and the temporal pattern, we found that participants were increasingly likely to report that the audiovisual sequences were synchronous. However, when we manipulated pitch and temporal predictability independently, the same effect did not occur. By altering the temporal density (items per second) of the sequences, we further determined that the predictability effect occurred only in temporally dense sequences: If the sequences were slow, participants' responses did not change as a function of predictability. We propose that reduced predictability affects synchrony judgments by reducing the effective pitch and temporal acuity in perception of the sequences.
\end{abstract}

Keywords Multisensory processing $\cdot$ Perceptual organization $\cdot$ Temporal processing

When we perceive objects in the environment around us, we often utilize more than one sense to identify, locate, and monitor what they are, where they are occurring, and their current state. The task of managing incoming sensation and

L. A. Cook $(\varangle) \cdot$ D. L. Van Valkenburg • D. R. Badcock

School of Psychology (M304), University of Western Australia,

35 Stirling Hwy,

Crawley 6009, Western Australia, Australia

e-mail: lauracook@graduate.uwa.edu.au determining how it should be organized into percepts is a computationally complex one, yet we accomplish it with ease, only rarely mismatching stimuli from differing sources. In the present article, will discuss some of the factors that contribute to this matching process in the temporal domain for hearing and vision - that is, the perception of synchrony. This process is not perfect: Participants routinely misjudge many types of stimuli as being temporally coincident when up to several hundred milliseconds apart (e.g., van Wassenhove, Grant, \& Poeppel, 2007). The ability to judge synchrony is important in determining how we structure our perceptual world, since simultaneity is one of the cues most utilized in deciding what information belongs with which object (Bregman, 1990).

Typically, multisensory interactions are investigated using situations where the incoming stimuli from each sense are conflicting - for instance, across space, time, or identity - often resulting in illusory percepts for one of the senses (e.g., McGurk \& MacDonald, 1976; Morein-Zamir, Soto-Faraco, \& Kingstone, 2003; Thurlow \& Jack, 1973). These illusory percepts provide information about how incoming sensation is processed and matched across space and time. Audiovisual interactions are amongst the most commonly described crossmodal illusions. For example, consider spatial ventriloquism illusions, in which auditory and visual items are perceived as occurring in the same location in space even though they may be some distance apart. This phenomenon is exemplified by a ventriloquist and dummy: We perceive the ventriloquist's voice to be coming from the dummy's mouth even though this isn't the case, because the visual stimulus is convincing enough to suggest that the voice should be localized there (see Vroomen, 2004, for a review).

Similar illusions occur across the time dimension between modalities in the temporal ventriloquism effect. If 
we present auditory and visual stimulation asynchronously, they still may be perceived as occurring at the same time; for instance, Slutsky and Recanzone (2001) showed that single flashes and tones or white noise bursts presented within 50-100 ms of each other would appear to be simultaneous. These effects have been shown across a variety of stimulus types, from single flashes and beeps through to more complex sequences (e.g., Cook \& Van Valkenburg, 2009) and more naturalistic input such as speech, music, and object actions (such as repeatedly hitting an object, Dixon \& Spitz, 1980; Vatakis \& Spence, 2006).

When considering audiovisual synchrony judgments, we must consider that there are differences in the stimulus processing times based on the different speeds of sound and light, and different processing times for information from each modality both peripherally and cortically (Efron, 1970; King, 2005). These sources of variability result in the sensation from each stimulus being synchronously processed when they are approximately 10-15 m awaycloser than this and the auditory stimuli are processed earlier because of faster sensory transduction times, and further away the visual stimuli are processed earlier because of the slower speed of sound (Vroomen \& Keetels, 2010). Despite these factors, we can still easily pair the two sources of information, suggesting that the temporal ventriloquism effect may be due to there being a window within which the sensation from each modality, if matched in other ways (for example, semantically or spatially), will be considered to originate from the same source (Spence \& Squire, 2003).

This window, or the temporal range over which auditory and visual stimuli are likely to be integrated, appears to differ substantially depending on the type of stimuli used in the experiment. This difference is typically measured as the just noticeable difference-the delay between the senses during which the modalities are just perceived as asynchronous. It is relatively short for simple stimuli, in the order of tens of milliseconds (e.g., Hirsh, 1961, 20 ms; Zampini, Shore, \& Spence, 2003, 20-60 ms), whereas with more complex speech or music stimuli, it may be several hundred (e.g., Massaro, Cohen, \& Smeele, 1996, 150-250 ms; van Wassenhove et al., 2007, $200 \mathrm{~ms}$ ). These time periods extend beyond any variability that can be attributed to source distance or processing time alone. Previous authors have noted this discrepancy (see, e.g., Vatakis \& Spence, 2010 , for a review of synchrony perception in simple and complex stimuli) and have explicitly compared different types of stimuli to try to determine what types of auditory and visual stimuli are more likely to be perceived as simultaneous. Dixon and Spitz (1980) asked participants to view videos of a person reading or of a hammer hitting a nail and identify at which point the auditory track, which was slowly becoming asynchronous, was discernibly "out of synch" with the video. All participants identified asynchrony between the auditory and visual components more quickly for the hammer video than for the speech video. Similarly, Vatakis and Spence (2006) examined participants' perceptions of synchrony for music, speech, and object actions such as repeatedly smashing a TV with a hammer and hitting a can with a block of wood. They found that participants required a wider time interval between the auditory and visual stimuli to detect asynchrony in the music clips as compared with the speech clips, and similarly needed a wider interval for the speech stimuli than for the object action stimuli. The authors interpreted these differences as being due to differing levels of complexity in each type of stimulus.

Within speech stimuli, length of the stimulus and familiarity may have an effect on synchrony judgments (Vatakis \& Spence, 2010); however, there is no comprehensive definition of complexity that describes the factors that make speech and music stimuli computationally different to sequences of beeps and flashes. Determining these factors may help to account for the different temporal windows associated with each.

One factor that may be a core component of complexity is the temporal density of the stimulus, as was suggested by Fujisaki and Nishida (2007). They used pulse train sequences with unpredictable patterns, and showed that participants are less able to detect asynchrony as the presentation rate increases. After about five items per second, the task became substantially more difficult for the participants because the auditory and visual stimuli always appeared to be synchronous. However, this cannot solely account for what is meant when we use the term complexity, since temporal density is not the only difference between simple flashes and beeps and speech or music stimuli. The audiovisual perception of speech, and especially music (when unknown to the listener), requires the management of constantly varying input, which changes in terms of content, timing, and location. As such, a second suggestion as to why we are more likely to match the content across modalities in complex stimuli is that increased spatial, temporal, or pitch variability, or perhaps how predictable the sequences are, also contributes to this process.

In the studies reported in the present article, we manipulated the pitch and temporal predictability of audiovisual sequences to determine whether these contribute to perception of synchrony. Petrini, Russell, and Pollick (2009) showed that audiovisual synchrony judgments can be improved when participants have the ability to predict the timing of the incoming unimodal stimuli. They used both detailed and sparse biological motion stimuli of a drummer and compared the synchrony judgments of 
novices with those of experienced drummers. The authors found that the experienced participants were better able to perform the task than were novices in the sparse conditions, and they suggested that this was because of their superior ability to predict the timing occurrence of the auditory stimuli on the basis of what few visual elements remained when the stimuli became impoverished. All rhythmic stimuli carry predictive information, and as Vroomen and Keetels (2010) suggested, this may be why we appear to be better at making temporal judgments about them than about nonrhythmic stimuli. In the present study, we manipulate the amount of predictable information explicitly using sequences of tones that may or may not repeat within a trial.

Earlier work (Cook \& Van Valkenburg, 2009) showed that temporal ventriloquism could be seen between grouped auditory and visual sequences once they were organized intramodally; therefore, the sequences used in the present study incorporated flashes and tones that would be subject to auditory and visual grouping into separate high and low streams. Grouped auditory and visual stimuli were used (rather than single streams) because the separation between attended and background stimuli increases their similarity to ecological stimuli (such as detecting speech from a background of noise) where this grouping is constantly occurring and increases the complexity of the task. Since pilot testing indicated that the effect of predictability discussed in the present article occurs most strongly when participants find the task challenging, we chose these sequences to ensure adequate perceptual load.

In the case of auditory streaming, when using repeating sequences of tones across different pitch ranges (e.g., highlow-high-low, across different ranges, similar to those in Fig. 1), the tones may be heard as one or two streams, depending on the separation in pitch between the tones and/ or the speed of the sequence. At slower speeds and with smaller pitch differences, the tones are heard as one stream rising and falling; however, at faster speeds and at larger pitch differences, the sequence perceptually splits into a high stream and a low stream, with participants no longer being able to reconcile the order of tones across the two (Bregman, 1990). A similar phenomenon occurs with visual stimuli: When flashes are presented at two different locations, they may appear as a single light moving back and forth or as two lights flickering in place, depending on the distance between them and the speed of presentation (Anstis, Giaschi, \& Cogan, 1985; Bregman \& Achim, 1973). In the present experiments, the different groups within both the auditory and visual sequences were presented at a large spatial or pitch difference and at a rate that ensured that they split into two streams, following the guidelines suggested by van Noorden (1975) and Anstis et al. (1985). Since the high and low streams were easily separable, participants could readily attend to either the high or low streams, and as such, the participant's task was always to determine whether the high pitched tones were coincidental with the spatially high lights, or vice versa. Pitch and vertical height appear to have a natural crossmodal correspondence. For instance, it has been shown that speeded classification of vertical location is faster when accompanied by a congruently pitched tone (Evans \& Treisman, 2010); therefore, participants were asked to match high pitch with high space or low pitch with low space, depending on which region they had chosen to attend to. For both auditory and visual stimuli, participants can only actively attend to one stream at a time (Duncan, Martens, \& Ward, 1997); therefore, the participants were always processing both attended and unattended information (i.e., an attended stream and background noise).

In Experiment 1, we examined whether manipulating the temporal and pitch predictability of audiovisual sequences changes participants' synchrony judgments. The auditory and visual sequences ranged from repeating two high and two low sequences (across pitch for the tones, space for the lights) to being randomly distributed with no discernable pattern. ${ }^{1}$ Sequences were presented synchronously or with the auditory or visual sequence presented first at a range of temporal distances between the stimuli from each modality. Given that more complex audiovisual stimuli have generally been found to increase the likelihood of participants perceiving crossmodal synchrony (Dixon \& Spitz, 1980; Vatakis \& Spence, 2006), if predictability contributes to complexity, we expect that participants would be more likely to report that they perceived the auditory and visual sequences as synchronous at a wider range of temporal discrepancies in the less predictable conditions.

Participants were asked to make judgments of synchrony rather than temporal order judgments (TOJs) for several reasons. Primarily, since the aim was to determine when they would perceive synchrony, this task was more appropriate: As was discussed in van Eijk, Kohlrausch, Juola, and van de Par (2008), synchrony judgments and TOJs target different processes, and synchrony judgments are more stable when the intent is to measure whether synchrony is perceived. Additionally, TOJs could not be used with the type of stimuli described presently because with these sequences, when they are in a predictable order, auditory first and visual first judgments are both technically correct because of the repeating two high/two low pattern, especially when asynchrony is large.

\footnotetext{
${ }^{1}$ Note that predictability does not affect auditory streaming (French-St George \& Bregman, 1989): Participants perceive the high and low tones as coming from separate streams, even when the regularity of the sequence is changed.
} 


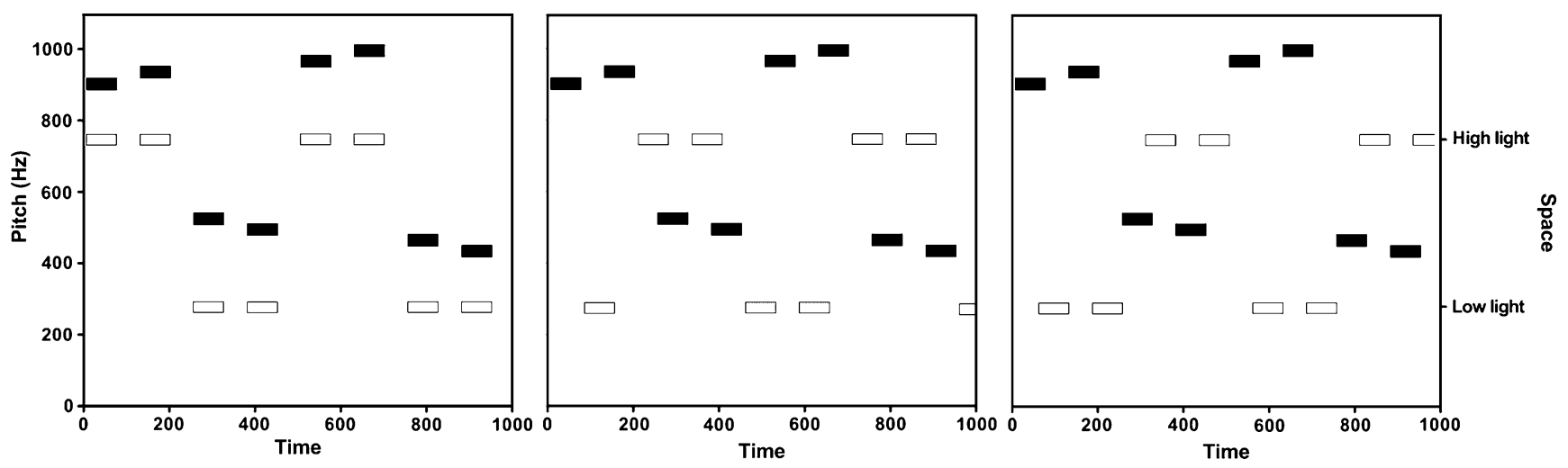

Fig. 1 The basic two-high/two-low auditory sequence when the audiovisual streams a were synchronous, b had an auditory lead of $200 \mathrm{~ms}$, and $\mathbf{c}$ had a visual lead of $200 \mathrm{~ms}$ (8-fixed condition). Filled rectangles represent tones, unfilled rectangles represent flashes

In Experiments 2 and 3, we attempted to determine whether either temporal or pitch predictability alone affects synchrony judgments, and in Experiment 4, we incorporated a manipulation of temporal density with conditions from the first experiment to examine whether this interacts with predictability.

\section{Experiment 1}

\section{Method}

Participants Eleven experienced observers volunteered to participate in the experiment as part of a course requirement (mean age $=21.9$ years, nine female; four additional participants completed the tasks but were not used in the final analysis, and are discussed in the results). Participants had completed at least 2 hours of similar crossmodal matching tasks prior to this experiment. All reported normal or corrected to normal vision. Participants gave their informed consent before participating in the study, and the experimental protocol was approved by the University of Western Australia's Human Research Ethics Committee.

Stimuli Both the auditory and visual sequences were based on the same two high/two low pattern shown in Fig. 1, repeated four times. Within the auditory sequence of sinusoidal tones, the high tones (T1, T2, T5, T6) were presented at $904 \mathrm{~Hz}, 934 \mathrm{~Hz}, 964 \mathrm{~Hz}$, and $994 \mathrm{~Hz}$ and the low tones (T3, T4, T7, T8) at $523 \mathrm{~Hz}, 493 \mathrm{~Hz}, 463 \mathrm{~Hz}$, and $433 \mathrm{~Hz}$. All of the tones had 10-ms linear onset and offset ramps and a duration of $70 \mathrm{~ms}$ followed by a $55-\mathrm{ms}$ silent interval (125 ms onset to onset). These pitches and durations were chosen so that the high and low tones would form separate auditory streams (at a rate of eight items per second; van Noorden, 1975). Because participants were able to attend to only one stream at a time, the effective speed of the stimuli within each stream was four tones per second (but two streams were always presented within the same trial). The tones were calibrated to equal loudness (approximately $70 \mathrm{~dB}$ ) using a Brüel and Kjær Type 4152 Artificial Ear and Brüel and Kjær 2260 Investigator Sound Level Meter, and were presented through Sennheiser HD590 headphones.

The visual stimuli were created by two $5-\mathrm{mm}$ green (570 nm) light-emitting diodes (LEDs; http://www.jaycar. com.au/productView.asp? ID $=$ ZD0170) that were vertically separated by $14^{\circ}$ of visual angle at a viewing distance of $75 \mathrm{~cm}$, connected to a PhidgetLED-64 board (www. phidgets.com) that was controlled by MaxMSP 4.5 software (cycling74.com). The visual sequence occurred in a similar pattern to that in Fig. 1, with the same stimulus and sequence durations as the auditory sequence. The high LED corresponded to each tone between 904 and $994 \mathrm{~Hz}$, and the low LED to those between 433 and $523 \mathrm{~Hz}$. The separation in space and time between the two lights ensured that there was no apparent motion; they appeared as two separate lights flickering in place (Anstis et al., 1985). The entire crossmodal sequence lasted approximately $4 \mathrm{~s}$ (125ms onset to onset time $\times 32$ items). ${ }^{2}$ Both the auditory and visual stimuli were controlled using the same MaxMSP 4.5 software running on Macintosh G3 computers.

There were five conditions within the experiment, each with differing levels of predictability. In each of the following conditions, complexity was operationalized as the proportion of the sequence that was randomly selected; that is, for each of the sequences, a proportion of the tones were fixed at the predictable values for tones T1-T8 described in the basic sequence above, whereas the remaining tones randomly filled any of the other positions without replacement. Because the eight tones were repeated

\footnotetext{
${ }^{2}$ It takes 2 to $3 \mathrm{~s}$ to organize incoming auditory information into streams (Anstis \& Saida, 1985); this sequence duration was chosen to ensure that streaming had time to occur before participants made their judgments.
} 
four times to form the entire sequence, we ensured that the random portions of the sequence were different for each repetition. There were equal numbers of unpredictable tones in both the high and low streams in all five conditions. The conditions were as follows:

0-fixed: All tones 1-8 random.

2-fixed: Two fixed tones; tones 1 and 3 fixed, remainder random.

4-fixed: Four fixed tones; 1, 2, 3, and 4 fixed, remainder random.

6-fixed: Six fixed tones; 1, 2, 3, 4, 5, and 7 fixed, remainder random.

8-fixed: All tones 1-8 fixed.

In the 8-fixed condition, the pitches ascended across the high stream and descended across the low stream, so it was possible to predict both the temporal pattern (two high/two low) and the pitch pattern (ascending or descending, depending on which stream was being attended to). The fewer fixed tones there were, the more the sequences deviated from the two high/two low pattern; therefore, the temporal pattern became less predictable and, similarly, the next pitch within the high or low range became less predictable as the ascending or descending order was lost.

The method of constant stimuli (MOCS) was employed, and within each of the five conditions, there were nine levels of asynchrony (stimulus onset asynchrony [SOA]) between the sounds and the lights ranging from auditory leading by $200 \mathrm{~ms}$ to visual leading by $200 \mathrm{~ms}$ in $50-\mathrm{ms}$ increments. In Fig. 1, examples of the timing of three SOAs are presented: the synchronous condition and the maximal visual and auditory leading conditions ( $\pm 200 \mathrm{~ms})$. To prevent an obvious delay at the start or end of the sequence in which the auditory or visual stream was present without the other, extra tones and flashes were added. If the delay was 100 or $150 \mathrm{~ms}$, one tone or flash was added, and if it was $200 \mathrm{~ms}$, two were added. This meant that the small delay that remained was not predictive of the overall delay in the sequence. ${ }^{3}$ Half of the sequences started with a high light/sound, half with a low light/sound.

Each of the nine audiovisual delays (MOCS levels) was presented 20 times, yielding 900 trials for each participant throughout the experiment $(9$ levels $\times 5$ conditions $\times 20$ ). Trials from all conditions and MOCS levels were presented randomly within each of five 20-min sessions (randomized trial to trial), which participants completed across $2-28$ days.

Procedure Participants observed the sequences of auditory and visual stimuli and were asked to report via keyboard

\footnotetext{
${ }^{3}$ Observers were advised that the beginning and end of the crossmodal sequence were not informative when making their judgments
}

whether the high and low tones were synchronous with the high and low lights (yes or no response). If unsure, they were instructed to respond that the streams were asynchronous. This helped to ensure that participants reported only that the sequences were synchronous when they had observed a convincing synchronous percept. After each response, they heard a 1-s mask of random MIDI instruments $(20 \times 50 \mathrm{~ms})$ after which the next trial began automatically. Before the experimental trials began, participants were presented with eight demonstration sequences at 0 and $\pm 200 \mathrm{~ms}$ (with feedback), and then were given a self-terminated set of practice trials to complete until they were comfortable with making synchrony judgments (without feedback). The practice trials were terminated after a maximum of $5 \mathrm{~min}$ across all participants. Participants were free to attend to either the high or the low stream at any time during the task. As such, it was not an explicitly manipulated condition, although informal participant reports indicated that most attended to the high stream for the majority of trials. Participants completed the trials at their own pace.

Previous research suggests that intramodal grouping (within audition or vision) occurs prior to crossmodal matching (Cook \& Van Valkenburg, 2009; Keetels, Stekelenburg, \& Vroomen, 2007) and that participants attend only to the high or low stream, not both (Duncan et al., 1997). Therefore, once unimodal grouping has occurred, participants will attend only to the high or low items and make their synchrony judgment within a stream. Thus, audiovisual pairing may not occur with the items that are closest to being concurrent across all stimuli, but rather with those that are closest within a stream.

Data analysis The proportion of "synchronous" responses for each of the nine time lags was calculated for each condition for each participant. A Gaussian curve ${ }^{4}$ was fitted to these means (such as in Fig. 2), resulting in five curves for each participant. There are three free parameters in these fits. The sigma parameter of the curve fits was taken as a representation of the width of the curve; wider curves indicate more "synchronous" responses at higher temporal discrepancies and therefore poorer ability to discriminate synchronous from asynchronous sequences. The mean of the curve fits (the $x$-value corresponding to the highest point in the curve) was used as a representation of the point of subjective simultaneity (PSS); this was the point at which the auditory and visual streams were perceived as maximally synchronous. The maximum $Y$-value was also

\footnotetext{
${ }^{4}$ Of the form $y=Y_{\max } e^{\left(-0.5^{*}((x-\mu) / \sigma)^{\wedge} 2\right)}$, where $Y_{\max }$ corresponds to the height of the curve, $\mu$ is the point of subjective simultaneity between audition and vision, and $\sigma$ is the standard deviation or a measure of the spread of the curve for the specific conditions.
} 


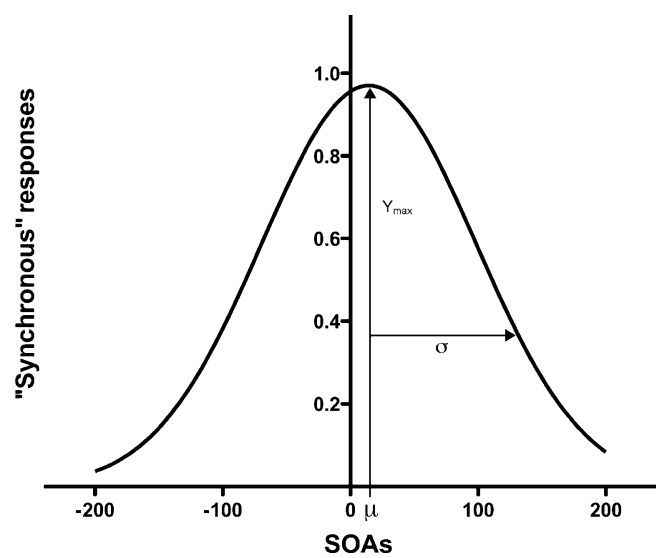

Fig. 2 Theoretical response curve. The $\sigma$ is taken as a measure of the width of the curve, $\mu$ as the point of subjective simultaneity, and $Y_{\max }$ as the amplitude of the curve

examined as a measure of amplitude to ensure that it did not vary across conditions.

\section{Results}

Four of the 15 initial participants were removed because either (a) Gaussian curves could not be fitted to their data in one or two of the conditions (three participants), ${ }^{5}$ or (b) their curve fits were unsatisfactory (one participant; in this case, all $R^{2}$ were less than .5). The mean $R^{2}$ across all curve fits for the experiment was .82 $(S D=.11)$.

The averaged responses across all SOAs are plotted in Fig. 3, for the 0 -fixed and 8 -fixed conditions. ${ }^{6}$ Of the three parameters estimated for these and the other conditions, two do not vary as a function of sequence predictability; oneway repeated measures ANOVAs showed that there was no significant effect of predictability on the amplitude of the curve, $F(4,40)=1.17, p=.34$, or on the PSS estimates [unfilled circles in Fig. $4 ; F(4,40)=1.00, p=.42$ ]. The mean sigma across participants did vary across the five predictability conditions [as shown by the filled circles in Fig. 4; $F(4,40)=4.04, p<.05, \eta_{\mathrm{p}}^{2}=.29$ ]. Bonferronicorrected post hoc $t$ tests showed that the 0 -fixed condition

\footnotetext{
${ }^{5}$ Postexperiment discussions with these participants suggested that they may have misunderstood the task or were unable to discriminate the pitches at all, rendering the task impossible. Their responses indicated that they either perceived the stimuli as synchronous across all SOAs in a condition (all proportions between 0.9 and 1; two participants) or were guessing throughout all of the conditions (all proportions were approximately 0.5 ).

${ }^{6}$ As can be seen in Fig. 4, there was high variation in the PSS values across participants, and there were also small variations in the amplitudes; as such, the curve appears wider and flatter at large SOAs than was seen for most individual participants when the data were averaged.
}

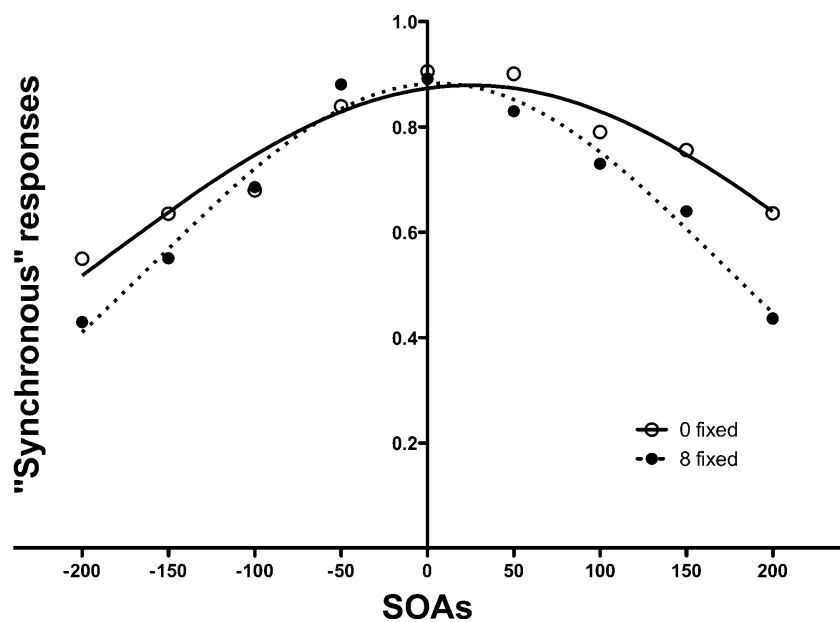

Fig. 3 Proportion of synchronous responses, averaged across observers for each of the SOAs in the 0 -fixed and 8-fixed conditions, with Gaussian curve fits $\left(R^{2}=.93\right.$ and .97 , respectively)

had a significantly higher mean sigma than the 8-fixed condition $(p<.05)$.

\section{Discussion}

The results of Experiment 1 show that when we decrease the predictability of both the pitch and temporal patterns, participants become worse at discriminating synchronous and asynchronous audiovisual sequences. This is consistent with what we would expect if predictability were a contributor to audiovisual complexity, given that the previous findings in relation to complexity show that participants are worse at judging synchrony when the stimuli are subjectively more complex (Vatakis \& Spence, 2010).

The method of response - that is, synchrony judgments versus temporal order judgments - may yield concern over participant bias; for example, when participants were

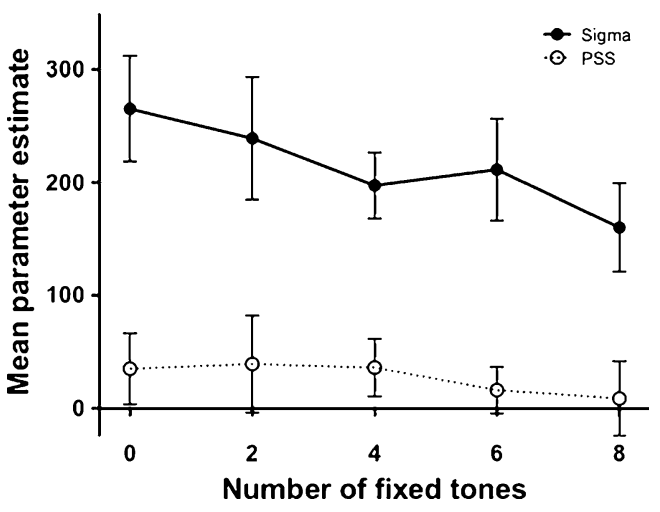

Fig. 4 Mean Gaussian sigmas and PSS estimates (in ms) for each condition for Experiment 1 (see text for details), with within-subjects 95\% confidence intervals (see Morey, 2008, for the method of calculation used here for within-subjects confidence intervals). Positive PSS estimates indicate visual leads 
confused, they may have been more inclined to respond that the stimuli were synchronous. However, this is unlikely to have affected our results for several reasons. First, the amplitudes of the curves were unaffected: If there were a bias in the more complex conditions, we would expect that the level of responding "synchronous" should change across the whole curve, creating a vertical shift. Secondly, because participants were explicitly asked to respond "asynchronous" if they were unsure about a particular crossmodal sequence, if the only difference between the groups was post-perceptual bias resulting from uncertainty, then we would expect the more random conditions to have lower proportions of synchrony judgments, as opposed to the increases we saw here.

It is possible that the increase in proportions of synchronous judgments with lowered predictability is primarily due to the manipulation of pitch or the manipulation of temporal predictability alone. In Experiments 2 and 3, we examined each of these components independently to determine whether the findings of Experiment 1 can be attributed to either component individually.

\section{Experiment 2}

In Experiment 2, we examined whether pitch predictability has an effect on synchrony judgments that is independent of temporal pattern predictability, by holding the twohigh/two-low pattern constant, but varying whether the next pitch within a stream was predictable (part of an ascending sequence in the high stream, descending in the low stream).

\section{Method}

Participants Eleven experienced observers, those who were able to complete the task in Experiment 1, took part in Experiment 2 as part of a course requirement (mean age $=$ 22.0 years, nine female). All reported normal or corrected-tonormal vision and hearing.

Stimuli The same basic auditory and visual sequences and procedure from Experiment 1 were used (Fig. 1). Instead of the randomization occurring to both the temporal order and pitch (i.e., to all items indiscriminately), in Experiment 2, only the pitch order of the tones within either the high or low stream was manipulated so that there was always a two high/two-low pattern, but the specific pitch that would occur next was not always predictable. The pitch order within the streams ranged from being entirely predictable (ascending in the high range, descending in the low, as in Fig. 1) to entirely random. This yielded four conditions. 0-fixed: Each high tone could be any of the four pitches from $904 \mathrm{~Hz}$ to $994 \mathrm{~Hz}$, and each low tone could be any of the four pitches from $523 \mathrm{~Hz}$ to $433 \mathrm{~Hz}$ (without replacement).

2-fixed: Tones 1 and 3 fixed; remainder randomly allocated without replacement.

4-fixed: 1, 2, 3, and 4 fixed; remainder randomly allocated.

8-fixed: All tones fixed as in Fig. 1.

As with the previous experiment, the high/low pattern of the flashes followed the same pattern as the high/low tones. Each of the four conditions was presented at each of the nine audiovisual time lags for 20 trials, resulting in 720 trials across four blocks of approximately $20 \mathrm{~min}$ each. All other methodological details were the same as in Experiment 1.

\section{Results}

The same Gaussian curve-fitting procedure was applied to the data for each participant in each of the four pitch predictability conditions as in Experiment 1 . The participants had an average $R^{2}$ value for the curve fit of $.77(S D=.05)$.

Of the three parameters estimated in each of the four pitch predictability conditions, none varied as a function of condition. One-way repeated measures ANOVAs showed that there was no significant effect of predictability on the amplitude of the curve, $F(3,30)=.82, p=.50$, on the PSS estimates [unfilled circles in Fig. $5 ; F(3,30)=$ $.84, p=.48$ ] or on the sigma estimates [filled circles in Fig. $5 ; F(3,30)=.25, p=.86]$.

\section{Discussion}

The results of Experiment 2 show that the effect found in Experiment 1 was likely not due to the effect of pitch

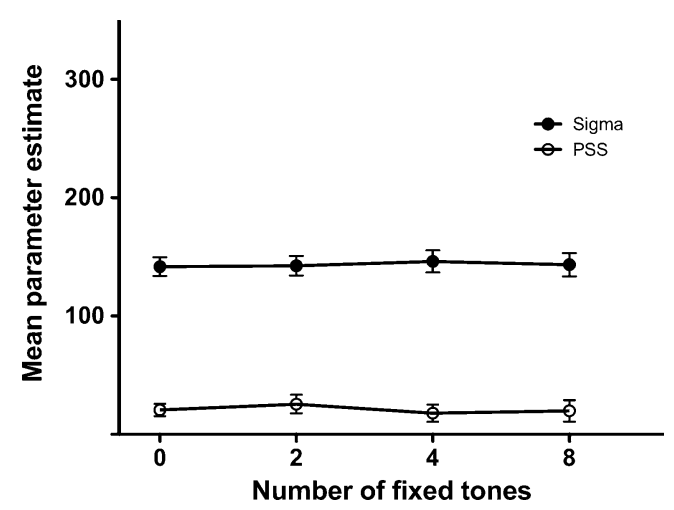

Fig. 5 Mean sigmas and PSS estimates (in ms) of the curves fitting proportion of synchronous responses to the SOA for Experiment 2, with within-subjects $95 \%$ confidence intervals. Positive PSS estimates indicate visual leads 
predictability alone, because when the temporal pattern was predictable (always two high/two low), there was no effect of varying the pitch predictability. Participants reported finding the task relatively simple in all conditions, and this is suggested in the Gaussian sigmas: The means for each condition were lower than those in Experiment 1 (Experiment 2 overall mean $=143.5 \mathrm{~ms}$; Experiment $1=214.7 \mathrm{~ms}$ ), and were most similar to those in the most predictable condition in Experiment 1 (8-fixed condition-160.2 ms).

\section{Experiment 3}

In Experiment 3, we examined whether the predictability of the temporal pattern has an effect on synchrony judgments that is independent of pitch predictability, by holding the pitch of the tones in the high and low streams constant.

\section{Method}

Participants Ten experienced observers, a subset of those who were able to complete Experiment 1, took part in the experiment as part of a course requirement (mean age $=$ 22.5 years, 8 female; two additional participants were removed before the final analysis). All reported normal or corrected-to-normal vision and hearing.

Stimuli Similar sequences and the same procedure from Experiment 1 were used. Instead of the randomization occurring across temporal order and pitch order (Experiment 1) or only within the pitches of an individual stream (Experiment 2), in Experiment 3, we primarily varied temporal predictability (i.e., whether the next tone was from the high or low stream, affecting the temporal pattern within each stream).

All of the high tones were presented at $904 \mathrm{~Hz}$, and all of the low tones were at $523 \mathrm{~Hz}$. In some trials, these were randomized across the eight tones so that whether the next pitch was high or low was unpredictable (and hence the temporal pattern within a stream was varied). The conditions were as follows:

0-fixed: Within each eight-tone section, there were four high and four low tones in any order.

2-fixed: Tone 1 was high and 3 was low, and the remaining positions could be high or low.

4-fixed: Tones 1 and 2 were high, 3 and 4 were low, and the remaining positions could be either high or low.

6-fixed: Tones 6 and 8 could be either high or low, and the remaining positions were fixed in their original pattern.

8-fixed: Tones fixed in a two-high/two-low pattern.

As with the previous experiments, the high/low pattern of the flashes followed the same pattern as the high/low tones. Each of the five conditions was presented 20 times within each of the nine time lags, yielding 900 trials. These were divided amongst five blocks, each taking 20 minutes. All other methodological details were the same as in Experiment 1 and 2.

\section{Results}

Two participants were removed from further analysis, since Gaussian curves could not be fit to their data in at least one condition. ${ }^{7}$ The remaining participants had an average $R^{2}$ value for the curve fit of $.81(S D=.11)$.

Of the three parameters estimated for each of the four temporal predictability conditions, none varied as a function of condition; one-way repeated measures ANOVAs showed that there was no significant effect of predictability on the amplitude of the curve, $F(4,32)=$ $1.94, p=.13$, on the PSS estimates [unfilled circles in Fig. $6 ; F(4,36)=1.16, p=.35]$, or on the sigma estimates [filled circles in Fig. $6 ; F(4,36)=.49, p=.74$ ].

\section{Discussion}

In Experiment 3, we showed that the effect of predictability found in Experiment 1 couldn't be accounted for by temporal predictability. Since neither temporal nor pitch predictability (as shown in Experiment 2) alone contribute to the effect, perhaps the perceptual system requires a certain amount of stress before the ability to make accurate synchrony judgments is affected. It is possible that many combinations of factor variation (e.g., pitch, spatial location, speed, temporal pattern) would cause a similar change in judgments through each independently increasing perceptual load, although it is also possible that some factors (or combinations of them) would be more influential than others with regard to this process.

\section{Experiment 4}

Another factor that has been shown to affect synchrony judgments is temporal density: Participants become worse at the task as the density increases, and above approximately five items per second, these judgments are no longer possible (Fujisaki \& Nishida, 2007). If both temporal density and predictability affect the subjective complexity of a stimulus, then it would be useful to determine how the two factors interact, since thus far we have studied only the effect of predictability at four items per second (within a stream). In

\footnotetext{
${ }^{7}$ Both participants had conditions in which they responded that the audiovisual sequences were synchronous across all SOAs.
} 


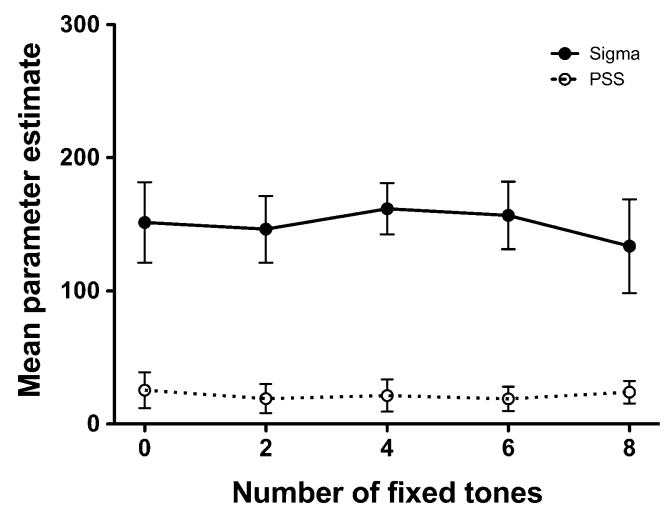

Fig. 6 Mean sigmas and PSS estimates (in ms) of the curves fitting proportion of synchronous responses to the SOA, across the five conditions, with within-subjects $95 \%$ confidence intervals. Positive PSS estimates indicate visual leads

Experiment 4, we examined the effect of temporal density changes in concert with predictability, using the 0 -fixed and 8 -fixed conditions from Experiment 1 at speeds of three, four or five items per second $(\mathrm{Hz})$ within a stream (higher temporal density corresponds to higher speeds). We expect that increased density would add another dimension of difficulty: If the tones are presented at a slower rate, there should be less effect of predictability (since lower density will allow better estimates of temporal position, and hence more accurate synchrony judgments, as was shown by Fujisaki \& Nishida, 2007). Conversely, if they are presented at a faster rate, the effects of randomness should increase, since the added speed increases the difficulty of making accurate temporal judgments, and judgments have been shown to be impaired at a $5-\mathrm{Hz}$ presentation rate.

\section{Method}

Participants Fifteen observers (who had not participated in Experiment 1-3) took part in the experiment as part of a course requirement (mean age $=23.1$ years, 11 female). All reported normal or corrected-to-normal vision and hearing. All but four participants had completed at least $1.5 \mathrm{hr}$ of similar crossmodal matching tasks prior to this experiment.

Stimuli The 0-fixed and 8-fixed conditions from Experiment 1 were used, crossed with three average temporal rates$3 \mathrm{~Hz}, 4 \mathrm{~Hz}$, and $5 \mathrm{~Hz}^{8}$ (which vary temporal density)resulting in six conditions. Items occurred every $166 \mathrm{~ms}$ in the $3-\mathrm{Hz}$ conditions, $125 \mathrm{~ms}$ (as in Experiment 1) in the 4-Hz conditions or $100 \mathrm{~ms}$ in the $5-\mathrm{Hz}$ conditions. Tone duration remained at $70 \mathrm{~ms}$ across all sequences, and the total

\footnotetext{
${ }^{8}$ The 3-, 4-, and 5-Hz presentation rates are within either the high or low stream, not across both. As was described in Experiment 1, participants could attend only to one stream at a time, so the presentation rates are denoted by the rate within one stream.
}

duration was $4,800 \mathrm{~ms}$ for the $3-\mathrm{Hz}$ conditions, $4,000 \mathrm{~ms}$ for the $4-\mathrm{Hz}$ conditions, and 3,200 $\mathrm{ms}$ for the $5-\mathrm{Hz}$ conditions.

Each of the six conditions was presented 20 times within each of the nine time lags, yielding 1,080 trials. These were divided amongst six blocks, each taking approximately $20 \mathrm{~min}$. All other methodological details were the same as in the previous experiments.

\section{Results}

As in previous experiments, Gaussian curves were applied to the means across the SOAs for each condition. The mean $R^{2}$ value across the conditions was $.83(S D=.10)$.

Means for each temporal density and predictability condition are displayed in Fig. 7. Mean sigmas of the Gaussian curve fits for each condition were higher for random sequences than for predictable sequences, and were higher for greater temporal densities. Figure 8 shows the averaged response curves across participants for the random and predictable conditions at $5-\mathrm{Hz}$ temporal density.

Of the three parameters estimated in each of the four pitch predictability conditions, two did not vary as a function of predictability or temporal density. Two-way repeated measures ANOVAs with these variables showed that there was no significant effect of predictability or of temporal density on the amplitude of the curve, $F(1,14)=$ $.13, p=.72$, and $F(2,28)=.84, p=.45$, respectively, or on the PSS estimates [unfilled circles in Fig. $6 ; F(1,14)=$ $2.05, p=.17$, and $F(2,28)=1.98, p=.16$ respectively]. There was also no interaction between predictability and temporal density in regard to the amplitudes, $F(2,28)=$ $1.44, p=.27$, or PSS estimates, $F(2,28)=.762, p=.48$.

However, both of these factors did affect sigma estimates: There was a main effect of predictability, $F(1$,

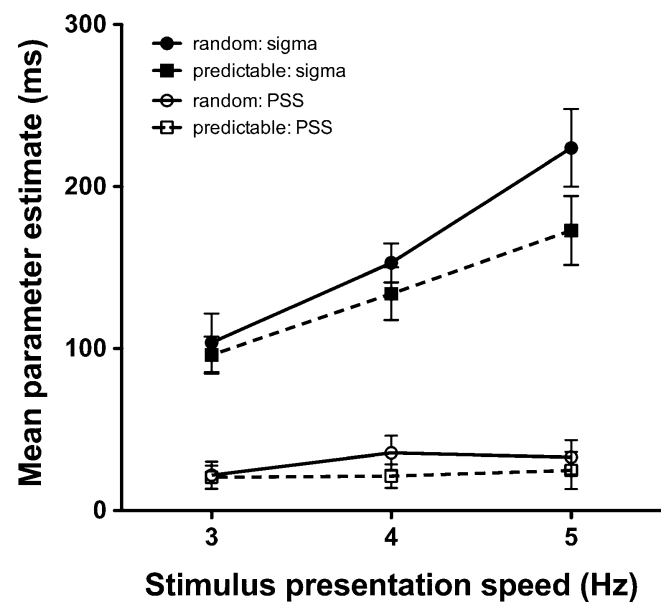

Fig. 7 Mean sigmas and PSS estimates (in ms) of the curves fitting proportion of synchronous responses to the SOA, with within-subjects 95\% confidence intervals. Positive PSS values indicate visual leads 


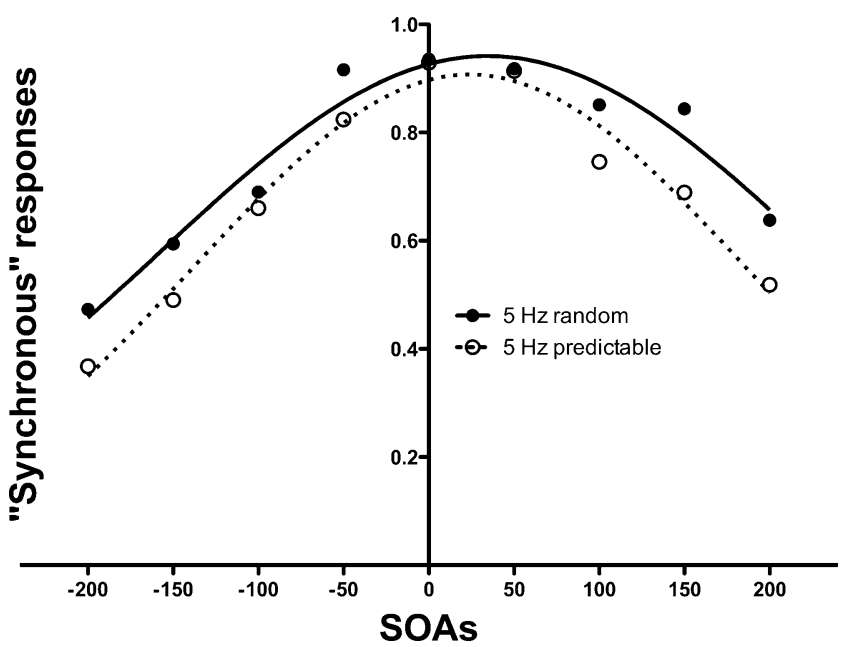

Fig. 8 Proportion of synchronous responses, averaged across observers for each of the SOAs in the random and predictable conditions at 5 -Hz temporal density, with Gaussian curve fits

14) $=4.78, p<.05, \eta_{\mathrm{p}}^{2}=.25$, and a main effect of temporal density, $F(2,28)=63.06, p<.001, \eta_{\mathrm{p}}^{2}=.82$, as well as an interaction between the two, $F(2,28)=4.06, p<.05$, $\eta_{\mathrm{p}}^{2}=.23$. Bonferroni-corrected post hoc $t$ tests indicate that this is because although there was a difference between the random and predictable sequences at a $4-\mathrm{Hz}$ or $5-\mathrm{Hz}$ temporal density $(p<.05)$, there was no difference when the temporal density was $3 \mathrm{~Hz}$.

\section{Discussion}

The results from Experiment 4 show that temporal density and predictability both contribute to the way we perceive audiovisual synchrony in sequences. There was also an interaction between the two: At relatively slow speeds ( $3 \mathrm{~Hz}$ within a stream), participants were equally able to detect asynchrony regardless of whether the sequence was predictable or random; however, as the sequences sped up, participants did better when the sequences were predictable.

\section{General Discussion}

These results show that there are several contributing factors to a participant's ability to detect temporal asynchrony between auditory and visual sequences. The results of Experiment 4 are consistent with the findings of Fujisaki and Nishida (2007): Increased temporal density reduces the ability to make judgments about asynchrony. Just as the effects of pitch and temporal predictability occurred only when they were both present within a sequence in Experiment 1, it is possible that increased temporal density adds another dimension to be monitored, and is therefore another factor that can add to the load of the perceptual system.
The effect of predictability increases when the items of a sequence are occurring relatively rapidly (see the increased difference between the random and predictable lines in Fig. 7 when the rate is $5 \mathrm{~Hz}$ ). There is also a difference between the influence of complexity in Experiment 1 and Experiment 4. Although there was no systematic difference between the participants in these two experiments (no difference in amount of experience, age, or obvious reasons for differential motivation), the mixture of different temporal rates and of individual differences between participants (often substantial in these kinds of tasks; see Spence \& Squire, 2003, for a discussion) could both be having an impact here. However, the impact of predictability on synchrony estimates is in the same direction in both studies.

In Experiment 11 and 4, we see changes in the sigma estimates of the curves, but not in the PSS estimates. This was to be expected: For the PSS values to be shifted, the factors would need to shift all of the auditory (or visual) temporal percepts earlier or later (not both). There is no reason to expect that varying predictability or temporal density would change estimates all in one direction, unlike factors such as distance (King, 2005) or possibly attention (Spence, Shore, \& Klein, 2001). Rather, predictability and temporal density may be affecting accuracy of temporal estimates (in both temporal directions) or increasing the evidence that the auditory and visual streams should be matched, both of which make the sequences appear more synchronous at higher SOAs.

We have a limited capacity to attend to and remember incoming perceptual objects (Lavie \& Tsal, 1994), and increasingly complex items - at least in the visual domain - are known to be more taxing to working memory (Eng, Chen, \& Jiang, 2005). It may therefore be only once the load becomes high-because there are many variables changing in rapid succession - that it becomes more difficult to monitor the input and make accurate judgments of temporal, spatial, and pitch positions, and therefore determine whether items should be matched crossmodally. This is consistent with what we have found across the experiments: Changing either pitch or temporal predictability alone has no effect, but when combined (and when temporal density is also changed), there is a change in participants' responses. Effectively, this increased perceptual loading may be acting to reduce the acuity for the incoming information and therefore change the accuracy of the estimated temporal boundaries of each item, increasing the likelihood of perceived overlap and making it more likely that items are matched erroneously across time, since mismatches are not detected.

It is also possible that increased numbers of unexpected temporal changes within each stream provides evidence for the auditory and visual streams to be matched if the pattern of changes in each is similar and within a small temporal 
interval. This may increase the likelihood that the two streams appear to "belong together," known as the unity assumption. Welch and Warren (1980) hypothesized that a belief that auditory and visual items belong together increases the chances that observers succumb to temporal and crossmodal illusions, raising the probability that they are perceived as a single event. There is evidence for an effect of semantic congruency, which supports the unity assumption, using speech stimuli. For example, Vatakis and Spence (2007) found that participants were worse at making temporal order judgments between sound and vision for speech video when the gender was matched across modalities as opposed to when one was male and one female. They interpreted this finding to be due to the mismatched stimuli being less susceptible to temporal ventriloquism since they were less likely to originate from the same source. Perhaps the computer-generated sequences used in the experiments detailed in the present article may be subject to a similar effect, since increasing the amount of transitions occurring in concert across both modalities may increase the belief that the auditory and visual sequences belong together. These increased congruent transitions occurred in the sequences because of both increasing randomness and increasing temporal density, and both of these factors had an effect. This result is consistent with the types of stimuli generally considered "complex" in previous literature, such as speech and music, since both have substantial numbers of transitions. This may be the underlying reason that more complex stimuli are more likely to be perceived as synchronous - because there is an increasing amount of rapid variations in sensation from each modality that are matched as the complexity increases.

The common factors described in the present article indicate that there may be a method to quantify the difference between single beeps and flashes, and natural stimuli in terms of complexity. The sigmas presented here are large (100-240 ms), indicating that across the predictability variants, participants were quite poor at making judgments with these kinds of stimuli. The possible similarities between these complex sequences and speech and music were mentioned previously, and indeed, it appears that participants match the auditory and visual streams across a similar range of asynchrony. As such, the factors described in the present article-increased levels of unpredictable information and the temporal density of the stimulus - may be a useful way to consider the differences between types of stimuli in a form that can be quantitatively measured. This suggests that when modeling crossmodal synchrony perception, we must consider the predictability of the incoming streams. Models of crossmodal interaction have often utilized maximum-likelihood estimation; that is, if the information from each modality is independent (with regard to position, timing, identity, etc.), the final estimate of position, timing, or identity is a weighted average of the two, weighted according to the reliability of the information contained in each (e.g., Alais \& Burr, 2004). The effect of predictability could be integrated into such models by increasing the variability of the unimodal estimates so that reduced ability to predict the incoming stimulus equates to wider distributions for the estimates of temporal (or spatial/ pitch) positions. Future research will examine spatial predictability, because this will help us to determine whether spatial factors-traditionally considered to be visually driven - would have an impact on synchrony judgments in a way similar to that of pitch predictability.

The experiments in the present article enable further understanding of the factors that contribute to the differences between simple auditory and visual stimuli and those that are more complex, such as speech and music. Both temporal density and the predictability of the sequence affect our ability to judge synchrony between auditory and visual streams, but this seems to occur only once the amount of varying information becomes substantial: We seem to have some "complexity threshold" that must be surpassed before judgments are affected.

Author Note Portions of the present research were presented at the 11th Annual Meeting of the International Multisensory Research Forum in Liverpool, UK, and at the 37th Australian Experimental Psychology Conference in Melbourne, Australia. This work was supported in part by a postgraduate scholarship to L.C. from the School of Psychology, University of Western Australia.

\section{References}

Alais, D., \& Burr, D. (2004). The ventriloquist effect results from near-optimal bimodal integration. Current Biology, 14, 257-262.

Anstis, S., Giaschi, D., \& Cogan, A. I. (1985). Adaptation to apparent motion. Vision Research, 25, 1051-1062.

Anstis, S., \& Saida, S. (1985). Adaptation to auditory streaming of frequency-modulated tones. Journal of Experimental Psychology. Human Perception and Performance, 11, 257-271.

Bregman, A. S. (1990). Auditory scene analysis. London: MIT Press.

Bregman, A. S., \& Achim, A. (1973). Visual stream segregation. Perception \& Psychophysics, 13, 451-454.

Cook, L. A., \& Van Valkenburg, D. L. (2009). Audio-visual organisation and the temporal ventriloquism effect between grouped sequences: Evidence that unimodal grouping precedes crossmodal integration. Perception, 38, 1220-1233.

Dixon, N. F., \& Spitz, L. (1980). The detection of auditory visual desynchrony. Perception, 9, 719-721.

Duncan, J., Martens, S., \& Ward, R. (1997). Restricted attentional capacity within but not between sensory modalities. Nature, 387 , $808-811$.

Efron, R. (1970). The minimum duration of a perception. Neuropsychologia, 8, 57-63.

Eng, H., Chen, D., \& Jiang, Y. (2005). Visual working memory for simple and complex visual stimuli. Psychonomic Bulletin \& Review, 12, 1127-1133.

Evans, K., \& Treisman, A. (2010). Natural cross-modal mappings between auditory and visual features. Journal of Vision, 10, 1-12. 
French-St George, M., \& Bregman, A. S. (1989). Role of predictability of sequence in auditory stream segregation. Perception \& Psychophysics, 46, 384-386.

Fujisaki, W., \& Nishida, S. (2007). Feature-based processing of audiovisual synchrony perception revealed by random pulse trains. Vision Research, 47, 1075-1093.

Hirsh, I. J. (1961). Perceived order in different sense modalities. Journal of Experimental Psychology, 62, 423-432.

Keetels, M., Stekelenburg, J., \& Vroomen, J. (2007). Auditory grouping occurs prior to intersensory pairing: Evidence from temporal ventriloquism. Experimental Brain Research, 180, 449456.

King, A. J. (2005). Multisensory integration: Strategies for synchronization. Current Biology, 15, 339-341.

Lavie, N., \& Tsal, Y. (1994). Perceptual load as a major determinant of the locus of selection in visual attention. Perception \& Psychophysics, 56, 183-197.

Massaro, D. W., Cohen, M. M., \& Smeele, P. M. T. (1996). Perception of asynchronous and conflicting visual and auditory speech. Journal of the Acoustical Society of America, 100, 1777-1786.

McGurk, H., \& MacDonald, J. (1976). Hearing lips and seeing voices. Nature, 264, 746-748.

Morein-Zamir, S., Soto-Faraco, S., \& Kingstone, A. (2003). Auditory capture of vision: Examining temporal ventriloquism. Cognitive Brain Research, 17, 154-163.

Morey, R. D. (2008). Confidence intervals from normalized data: A correction to Cousineau (2005). Tutorial in Quantitative Methods for Psychology, 4, 61-64.

Petrini, K., Russell, M., \& Pollick, F. (2009). When knowing can replace seeing in audiovisual integration of actions. Cognition, 110, 432-439.

Slutsky, D. A., \& Recanzone, G. H. (2001). Temporal and spatial dependency of the ventriloquism effect. Neuroreport, 12, 7-10.

Spence, C., Shore, D. I., \& Klein, R. M. (2001). Multisensory prior entry. Journal of Experimental Psychology. General, 130, 799-832.
Spence, C., \& Squire, S. (2003). Multisensory integration: Maintaining the perception of synchrony. Current Biology, 13, 519-521.

Thurlow, W. R., \& Jack, C. E. (1973). Certain determinants of the "ventriloquism effect. Perceptual and Motor Skills, 36, 1171-1184.

van Eijk, R. L. J., Kohlrausch, A., Juola, J. F., \& van de Par, S. (2008). Audiovisual synchrony and temporal order judgments: Effects of experimental method and stimulus type. Perception \& Psychophysics, 70, 955-968.

van Noorden, L. P. A. S. (1975). Temporal coherence in the perception of tone sequences. (Unpublished doctoral dissertation). Institute for Perceptual Research.

van Wassenhove, V., Grant, K. W., \& Poeppel, D. (2007). Temporal window of integration in auditory-visual speech perception. Neuropsychologia, 45, 598-607.

Vatakis, A., \& Spence, C. (2006). Audiovisual synchrony perception for music, speech, and object actions. Brain Research, $1111,134-142$

Vatakis, A., \& Spence, C. (2007). Crossmodal binding: Evaluating the "unity assumption" using audiovisual speech stimuli. Perception \& Psychophysics, 69, 744-756.

Vatakis, A., \& Spence, C. (2010). Audiovisual temporal integration for complex speech, object-action, animal call and musical stimuli. In M. J. Naumer \& J. Kaiser (Eds.), Multisensory object perception in the primate brain (pp. 95-122). New York: Springer.

Vroomen, J. (2004). Perceptual effects of cross-modal stimulation: Ventriloquism and the freezing phenomenon. In G. Calvert, C. Spence, \& B. Stein (Eds.), The handbook of multisensory processes (pp. 141-150). Cambridge: MIT Press.

Vroomen, J., \& Keetels, M. (2010). Perception of intersensory synchrony: A tutorial review. Attention, Perception, \& Psychophysics, 72, 871-884.

Welch, R. B., \& Warren, D. H. (1980). Immediate perceptual response to intersensory discrepancy. Psychological Bulletin, 88, 638-667.

Zampini, M., Shore, D. I., \& Spence, C. (2003). Audiovisual temporal order judgments. Experimental Brain Research, 152, 198-210. 